\title{
Narrow-escape-time problem: The imperfect trapping case
}

\author{
Félix Rojo, ${ }^{1}$ Horacio S. Wio, ${ }^{2}$ and Carlos E. Budde ${ }^{1}$ \\ ${ }^{1}$ Facultad de Matemática, Astronomía y Física, Universidad Nacional de Córdoba, Ciudad Universitaria, 5000 Córdoba, Argentina \\ ${ }^{2}$ Instituto de Física de Cantabria, Universidad de Cantabria and CSIC, E-39005 Santander, Spain \\ (Received 8 June 2012; revised manuscript received 14 August 2012; published 5 September 2012)
}

\begin{abstract}
We present a master equation approach to the narrow escape time (NET) problem, i.e., the time needed for a particle contained in a confining domain with a single narrow opening to exit the domain for the first time. We introduce a finite transition probability, $v$, at the narrow escape window, allowing the study of the imperfect trapping case. Ranging from 0 to $\infty, v$ allowed the study of both extremes of the trapping process: that of a highly deficient capture and situations where escape is certain ("perfect trapping" case). We have obtained analytic results for the basic quantity studied in the NET problem, the mean escape time, and we have studied its dependence in terms of the transition (desorption) probability over (from) the surface boundary, the confining domain dimensions, and the finite transition probability at the escape window. Particularly we show that the existence of a global minimum in the NET depends on the "imperfection" of the trapping process. In addition to our analytical approach, we have implemented Monte Carlo simulations, finding excellent agreement between the theoretical results and simulations.
\end{abstract}

DOI: 10.1103/PhysRevE.86.031105

PACS number(s): 05.40.Fb

\section{INTRODUCTION}

The time needed for a particle contained in a confining domain with a single small opening to exit the domain for the first time, usually referred to as the narrow-escape-time (NET) problem (see [1] and references therein), finds a prominent place in many domains and fields. For instance, in cellular biology, it is related to the random time needed by a particle released inside a cell to activate a given mechanism on the cell membrane [2-4]. Generally speaking, the NET problem is part of so-called intermittent processes, which are used to explain scenarios ranging from animal search patterns [5], through the solutions or melts of synthetic macromolecules [6,7], to the manufacture of self-assembled mono- and multilayers [8,9]. Since the work of Berg and Purcell [10], research in the NET problem area has experienced a steady growth over time and motivated a great deal of work [1,11-21].

In [19], we have introduced an analytical Markovian model that showed the impact of geometrical parameters and the interplay between surface and boundary paths in the studied confining domain, of a discrete and rectangular-shaped nature, for the perfect trapping case. By "perfect trapping" we refer to the particle's impossibility of return to the system; i.e., once the particle reaches the narrow opening the escape becomes certain. In that work we presented a phase diagram which showed that some combinations of the geometrical parameter and the transport mechanism were required for the existence of an optimal transport (a global minimum in the NET).

In this work we consider the same confining domain and the transport properties that we dealt with in Ref. [19]. However, we eliminate the assumption of perfect escape by introducing a finite transition probability at the narrow escape window. It is well known that system descriptions through the "imperfect trapping case" (i.e., once in the trap site capture is not certain) are suitable whenever the surface contains "deep traps," capture and re-emission from a surface that contains sites with several internal states such as the "ladder trapping model," proteins with active sites deep inside its matrix, etc. [22-25].
The aim of this work is to study the influence of the "imperfection" in the passage through the escape window on the effective diffusion process and its effect on the NET problem. For that purpose we calculate the time required by the particle to escape from the system. In order to perform our research we exploit Dyson's theory [26] and the notions of absorption probability density (APD) [23]. Under the assumption of imperfect trapping, we have discovered some very interesting results. Particularly, we show that the existence of a global minimum in the NET depends on the existence of an imperfection in the trapping process.

The outline of this paper is as follows. In the following section we introduce some general results regarding imperfect trapping process as well as our model, and we provide the basic definitions and concepts. We also describe the proposed analytical approach and present the main results. Section III depicts several assorted illustrations for the mean escape time to the narrow opening for different configurations of the system through a comparison between our analytical framework and Monte Carlo simulations. In Sec. IV we discuss our conclusions and perspectives. Finally, in the Appendices we present the calculations indicated in Sec. II.

\section{ANALYTICAL APPROACH}

\section{A. Some general results regarding imperfect trapping}

Let us consider the problem of a walker making a random walk in some finite domain with a trap or sink present in the system. We will follow the walker evolution through the system considering the "unrestricted" or "homogeneous" conditional probability $P\left(\vec{s}, t \mid \vec{s}_{0}, t=0\right)$, that is, the probability that a walker is at $\vec{s}$ at time $t$ given it was at $\vec{s}_{0}$ at $t=0$. By "unrestricted" (or homogeneous) we identify a situation with no traps or sinks present in the system.

\section{Absorption probability density and mean absorption time}

We will consider a first-order trapping process of parameter $v$ [23]. That is, the entrance to the trap site is regulated at the 


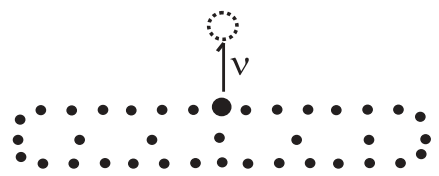

FIG. 1. Finite domain with a trap or sink present. The entrance to the trap site (empty circle) is regulated at the escape "window" by the transition rate $v$.

escape "window" by the transition rate $v$ (see Fig. 1). As we are interested in the imperfect trapping process, let us define $A\left(\vec{s}, t \mid \vec{s}_{0}, 0\right)$ as the APD through the site $\vec{s}$ at time $t$, given that the walker was at $\vec{s}_{0}$ at time $t=0$, i.e., $A\left(\vec{s}, t \mid \vec{s}_{0}, 0\right) d t$ gives the trapping probability of the walker, through $\vec{s}$, between $t$ and $t+d t$ given that it started at $t=0$ from $\vec{s}_{0}$. It is worth mentioning that the first-passage-time approach is not fully applicable since an "excursion" to the trapping site does not necessarily end the process. However, we show in the following lines that an interesting relation could be established between $A\left(\vec{s}, t \mid \vec{s}_{0}, 0\right)$ and $F\left(\vec{s}, t \mid \vec{s}_{0}, 0\right)$, the first-passage-time density (FPTD) through the site $\vec{s}$ at time $t$, given that the walker was at $\vec{s}_{0}$ at time $t=0$.

From now on we will denote the Laplace transform of a function $f(t)$ by its argument,

$$
\mathcal{L}\{f(t)\}=f(u)=\int_{0}^{\infty} e^{-u t} f(t) d t .
$$

The connection between the APD and the unrestricted conditional probability $P\left(\vec{s}, t \mid \vec{s}_{0}, t=0\right)$ could be obtained by resorting to the local inhomogeneity technique [23,27]. This approach in the Laplace domain gives

$$
A\left(\overrightarrow{0}, u \mid \vec{s}_{0}, t=0\right)=\frac{v P\left(\overrightarrow{0}, u \mid \vec{s}_{0}, t=0\right)}{1+v P(\overrightarrow{0}, u \mid \overrightarrow{0}, t=0)} ;
$$

this equation is also known as the generalized Siegert formula.

Let us make a brief digression regarding the relation between the APD and the FPTD. For this we rewrite Eq. (1) in the form

$$
A\left(\overrightarrow{0}, u \mid \vec{s}_{0}, t=0\right)=\frac{P\left(\overrightarrow{0}, u \mid \vec{s}_{0}, t=0\right)}{P(\overrightarrow{0}, u \mid \overrightarrow{0}, t=0)} \frac{v}{v+\frac{1}{P(\overrightarrow{0}, u \mid \overrightarrow{0}, t=0)}} .
$$

The connection between the FPTD and the "unrestricted" (homogeneous) conditional probability $P\left(\vec{s}, t \mid \vec{s}_{0}, t=0\right)$ is established (in the Laplace domain) through the "renewal" or Siegert approach [28],

$$
F\left(\overrightarrow{0}, u \mid \vec{s}_{0}, t=0\right)=\frac{P\left(\overrightarrow{0}, u \mid \vec{s}_{0}, t=0\right)}{P(\overrightarrow{0}, u \mid \overrightarrow{0}, t=0)} .
$$

We recognize in Eq. (2) the FPTD (3) and using the relation $F(\overrightarrow{0}, u \mid \overrightarrow{0}, t=0)=1-\Psi(\overrightarrow{0}, u) P(\overrightarrow{0}, u \mid \overrightarrow{0}, t=0)^{-1}$, where $\Psi(\vec{s}, \tau)$ is the probability that the walker remains at $\vec{s}$ until time $\tau$ since it arrived at $\vec{s}$ at time 0 (in the unrestricted case) [29], we rewrite Eq. (2) as

$$
\begin{aligned}
A\left(\overrightarrow{0}, u \mid \vec{s}_{0}, t=0\right)= & F\left(\overrightarrow{0}, u \mid \vec{s}_{0}, t=0\right) \frac{v}{\Psi(\overrightarrow{0}, u)^{-1}+v} \\
& \times \frac{1}{1-\frac{\Psi(\overrightarrow{0}, u)^{-1}}{\Psi(\overrightarrow{0}, u)^{-1}+v} F(\overrightarrow{0}, u \mid \overrightarrow{0}, t=0)} .
\end{aligned}
$$

The term $v\left(\Psi(\overrightarrow{0}, u)^{-1}+v\right)^{-1}$ in (4) gives the fraction of walkers that are trapped while $\Psi(\overrightarrow{0}, u)\left(\Psi(\overrightarrow{0}, u)^{-1}+v\right)^{-1}$ gives the ones that are not absorbed. Further considerations could be made regarding (4), and we write it as

$$
A\left(\overrightarrow{0}, u \mid \vec{s}_{0}, t=0\right)=\sum_{j=1}^{\infty} A_{j}\left(\overrightarrow{0}, u \mid \vec{s}_{0}, t=0\right),
$$

where

$$
\begin{aligned}
A_{j}\left(\overrightarrow{0}, u \mid \vec{s}_{0}, 0\right)= & F\left(\overrightarrow{0}, u \mid \vec{s}_{0}, 0\right)\left(\frac{F(\overrightarrow{0}, u \mid \overrightarrow{0}, 0) \Psi(\overrightarrow{0}, u)^{-1}}{\Psi(\overrightarrow{0}, u)^{-1}+v}\right)^{j-1} \\
& \times\left(\frac{v}{\Psi(\overrightarrow{0}, u)^{-1}+v}\right)
\end{aligned}
$$

Notice that $A_{j}(\cdot)$ accounts for walkers that are absorbed in the $j$ visit $(j=2,3, \ldots)$ and not before; i.e., the walkers arrive for the first time at site $\overrightarrow{0}$ from $\vec{s}_{0}$ but these are not absorbed until they return to site $\overrightarrow{0}$ for the $(j-1)$ time (see Appendix A).

The probability of being absorbed in the $j$ visit at site $\overrightarrow{0}$ is

$$
\begin{aligned}
\int_{0}^{\infty} & A_{j}\left(\overrightarrow{0}, t \mid \vec{s}_{0}, 0\right) d t \\
= & A_{j}\left(\overrightarrow{0}, u=0 \mid \vec{s}_{0}, 0\right)=\left(\frac{\Psi(\overrightarrow{0}, 0)^{-1}}{\Psi(\overrightarrow{0}, 0)^{-1}+v}\right)^{j-1} \\
& \times\left(\frac{v}{\Psi(\overrightarrow{0}, 0)^{-1}+v}\right),
\end{aligned}
$$

where $\Psi(\overrightarrow{0}, u=0)$ is the mean residence time at site $\overrightarrow{0}$ in the unrestricted system and we have used $F\left(\vec{s}, u=0 \mid \vec{s}_{0}, t=0\right)=$ 1 for a finite (unrestricted) system. As Eq. (7) shows, when $v \rightarrow 0$ independently of the $j$ value, we have no absorption, while in the limit $v \rightarrow \infty$ the absorption is certain in the first "visit" to the site (i.e., $j=1$ ).

The mean absorption time, i.e., the mean time until the walker is absorbed, is evaluated in terms of $A(\cdot)$ as

$$
\begin{aligned}
T & =\int_{0}^{\infty} t \sum_{\vec{s}_{0}} A\left(\overrightarrow{0}, t \mid \vec{s}_{0}, 0\right) g\left(\vec{s}_{0}\right) d t \\
& =-\left.\frac{\partial}{\partial u}\left\{\sum_{\vec{s}_{0}} A\left(\overrightarrow{0}, u \mid \vec{s}_{0}, 0\right) g\left(\vec{s}_{0}\right)\right\}\right|_{u=0},
\end{aligned}
$$

where $g\left(\vec{s}_{0}\right)$ denotes the probability density of initially finding the walker at a position $\vec{s}_{0}[28]$.

\section{B. The model}

For our model we consider the problem of a walker making a random walk in a finite rectangular $N \times(M+1)$ lattice (see Fig. 2). The surface is bounded in the $y$ direction where the walkers can move from $y=0$ to $y=M$, and periodic boundary conditions are assumed in the $x$ direction so $x$ and $x+N$ denote the same place in space. As we mentioned before, we follow the walker's evolution through the system 


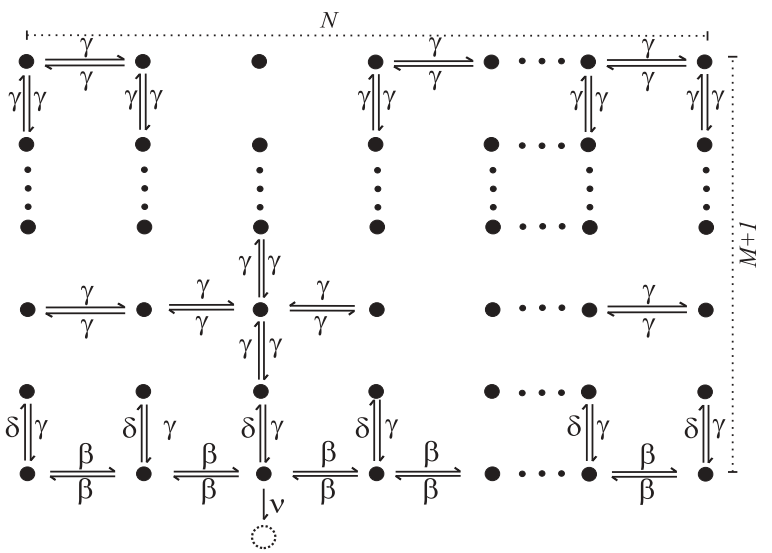

FIG. 2. Schematic transitions of the walker to or from the baseline and to or from a generic surface site. Notice that the entrance to the trap or escape site (empty circle) is regulated by the transition rate $v$; also notice that it could be reached both from the surface (with transition rate $\gamma$ ) and from the baseline (with transition rate $\beta$ ).

by considering the conditional probability $P\left(n, m, t \mid n_{0}, m_{0}, t=\right.$ $0) \equiv P(n, m, t)$, where $(n, m)$ are discrete coordinates in $(x, y)$ space. $P(n, m, t)$ satisfies the following master equations:

$$
\begin{aligned}
\dot{P}(n, 0, t)= & \gamma P(n, 1, t)-\delta P(n, 0, t)+\beta(P(n+1,0, t) \\
& +P(n-1,0,0, t)-2 P(n, 0, t)), \quad m=0 ; \\
\dot{P}(n, 1, t)= & \delta P(n, 0, t)-4 \gamma P(n, 1, t)+\gamma(P(n+1,1, t) \\
& +P(n-1,1, t)+P(n, 2, t)), \quad m=1 ; \\
\dot{P}(n, m, t)= & \gamma(P(n-1, m, t)+P(n+1, m, t) \\
& +P(n, m+1, t)+P(n, m-1, t)) \\
& -4 \gamma P(n, m, t), \quad 2 \leqslant m \leqslant M-1 ; \\
\dot{P}(n, M, t)= & \gamma(P(n-1, M, t)+P(n+1, M, t) \\
& +P(n, M-1, t))-3 \gamma P(n, M, t), \quad m=M,
\end{aligned}
$$

where $\gamma$ is the surface transition probability per unit time in the $x$ and $y$ directions, $\beta$ is the transition probability over the line $m=0$ in the $x$ direction, and $\delta$ is the desorption probability per unit time from the boundary line $m=0$.

We introduce the imperfect escape case by allowing a finite transition probability $(v)$ at the narrow escape window. Varying from 0 to $\infty, v$ allowed the study of both a deficient trapping and situations where escape is certain (i.e., the perfect trapping case).

In the following we will say that the walker "escapes" when it gets trapped or adsorbed, without the possibility of returning to the system. This terminology matches the one used in the NET area. Hence, adsorption $\rightarrow$ escape, and so on.

\section{Escape probability density}

We now make a brief comment regarding the escape probability density. Taking into account the parameters of our model, $\Psi(\overrightarrow{0}, 0)=(u+\delta+2 \beta)^{-1}$, we could write Eq. (7) as

$$
\begin{aligned}
\int_{0}^{\infty} A_{j}\left(\overrightarrow{0}, t \mid \vec{s}_{0}, 0\right) d t & =A_{j}\left(\overrightarrow{0}, u=0 \mid \vec{s}_{0}, 0\right) \\
& =\left(\frac{2 \beta+\delta}{2 \beta+\delta+v}\right)^{j-1}\left(\frac{v}{2 \beta+\delta+v}\right) .
\end{aligned}
$$

Notice that as $v$ grows $(\nu \gg 2 \beta+\delta)$ each $A_{j}$ becomes smaller except for $A_{1}$, with $A_{1} \rightarrow 1$; i.e., the escape is certain in the first visit. However, when $v \ll 2 \beta+\delta$, the probability of escape $A(\cdot)$ has contributions from each $j$ visit. This can best be understood considering

$$
\left.\frac{A_{j}(\cdot)}{A_{j+1}(\cdot)}\right|_{u=0}=1+\frac{v}{2 \beta+\delta} \quad(j \neq 1),
$$

which gives the relative contribution of successive terms in (5). When $v$ gets smaller the contribution is spread all over $j$ values, as Eq. (11) shows. In contrast, each $A_{j} \rightarrow 0(j \neq 1)$ as $v$ grows, concentrating all the probability in $A_{1}$.

\section{Mean escape time}

Following the ideas exposed in [19] and by resorting to the matrix formalism and Dyson's procedure [26] we were able to obtain the probability $P\left(n, m, t \mid n_{0}, m_{0}, t=0\right)$ (in the Fourier-Laplace space), which is the building block for the mean escape time (MET). For the detailed calculation see Appendix B.

We will denote the (finite) Fourier transform by its argument, as we did in the Laplace transform case. Thus for example the transform on a coordinate, say $x$, would read

$$
\begin{aligned}
P\left(k, m, t \mid n_{0}, m_{0}, 0\right) & \equiv \mathcal{F}\left\{P\left(n, m, t \mid n_{0}, m_{0}, 0\right)\right\} \\
& =\sum_{n=0}^{N-1} e^{i k n} P\left(n, m, t \mid n_{0}, m_{0}, 0\right) .
\end{aligned}
$$

From $P\left(k, m, u \mid n_{0}, m_{0}, t=0\right)$ (obtained in the Fourier-Laplace space), the probability that a walker is on the surface at site $(n, m)$ at time $t$ given it was at $\left(n_{0}, m_{0}\right)$ at $t=0$, $P\left(n, m, t \mid n_{0}, m_{0}, t=0\right)$, is derived by using the inverse Laplace transform on $u$ and the inverse Fourier transform on $k$ (for the $x$ coordinate) for each $[\mathbb{P}(k, u)]_{m, m_{0}}$. However, as we are interested in the calculation of (8), we only need to perform the inverse Fourier transform on $P\left(0,0, u \mid n_{0}, m_{0}, t=0\right)$; i.e., we need the elements $\mathcal{F}^{-1}\left\{[\mathbb{P}(k, u)]_{0, m_{0}}\right\}$. We obtain for $[\mathbb{P}(k, u)]_{0, m_{0}}$

$$
[\mathbb{P}(k, u)]_{0, m_{0}}=\frac{\eta^{m_{0}}+\eta^{\tilde{M}-m_{0}}}{\delta(1-\eta)\left(1-\eta^{\tilde{M}-1}\right)+\left(u-A_{1}(k)\right)\left(1+\eta^{\tilde{M}}\right)},
$$

where $\eta=1+\left(\tilde{u}-\sqrt{\tilde{u}^{2}+4 \gamma \tilde{u}}\right) / 2 \gamma, \tilde{M}=2 M+1$, and $\tilde{u}=$ $u-A(k)$. The inverse Fourier transform on $[\mathbb{P}(k, u)]_{0, m_{0}}$ is carried out in the following way:

$$
P\left(0,0, u \mid n_{0}, m_{0}, 0\right)=\frac{1}{N} \sum_{q=0}^{N-1} e^{i \frac{2 \pi n_{0} q}{N}}\left[\mathbb{P}\left(\frac{2 \pi q}{N}, u\right)\right]_{0, m_{0}} .
$$


Thus we have obtained the required expression for the calculation of the MET through the narrow escape window and it only remains to choose the initial distribution. We now evaluate the MET for a walker with a uniform initial distribution on the baseline $(y=0)$. This means that the initial distribution is given by $g(n, m)=\left(1-\delta_{n, 0}\right) \delta_{m, 0} /(N-1)$. Notice that we explicitly exclude the possibility of having a walker at $(0,0)$ at $t=0$ [30]. In this way we obtain

$$
T=N\left[\frac{M}{\gamma}+\frac{1}{\delta}\right]\left\{\frac{\delta}{v}+\frac{\delta}{N-1} \sum_{q=1}^{N-1}\left[\mathbb{P}\left(\frac{2 \pi q}{N}, u=0\right)\right]_{0,0}\right\} .
$$

We make some comments regarding Eq. (14) which constitutes one of our main results. Notice that (14) adequately provides the limits of the perfect escape case, $v \rightarrow \infty$ (obtained in [19]) and the no escape window, $v \rightarrow 0, T \rightarrow \infty$. Observe that, as is commented in [1], for a perfect escape case, $T$ could be expressed in the following way:

$$
\begin{aligned}
T= & \frac{N M}{\gamma}\left[\frac{\delta}{v}+\delta \frac{\sum_{q}\left[\mathbb{P}\left(\frac{2 \pi q}{N}, u=0\right)\right]_{0,0}}{N-1}\right] \\
& +\frac{N}{2 \beta}\left[\frac{2 \beta}{v}+2 \beta \frac{\sum_{q}\left[\mathbb{P}\left(\frac{2 \pi q}{N}, u=0\right)\right]_{0,0}}{N-1}\right],
\end{aligned}
$$

or $T=T_{\text {surface }}+T_{\text {line }} ;$ (15) provides an interesting physical insight into the problem. Simply notice how the mean escape time is constructed from the mean duration of surface excursions and the mean duration of border or line excursions [first and second terms of (15), respectively].

\section{The existence of a minimum in $T$}

The mean time $T$ could be enhanced with respect to $\delta$ provided we are able to find $\delta^{*}=\delta^{*}(\beta, \gamma, \nu, N, M)$-the optimal desorption probability - that satisfies

$$
\left.\frac{\partial T}{\partial \delta}\right|_{\delta=\delta^{*}}=\frac{N M}{\gamma \nu}+\frac{N}{N-1} \sum_{q=1}^{N} \frac{M \gamma^{-1} 2 \beta \alpha_{2}-\alpha_{1}}{\left(\alpha_{1} \delta^{*}+\alpha_{2} 2 \beta\right)^{2}}=0,
$$

where the $\alpha_{i}=\alpha_{i}(q, N, M)$ for $i=1,2$ are defined in Appendix C. Notice that (16) defines an implicit equation for $\delta^{*}$, which, although we could not solve, provided us with some general conclusions as it approaches certain limits. Consider first $\beta \rightarrow 0$, with the other parameters held fixed. In this case (a finite value for) $\delta^{*}$ exists whenever we have a finite escape probability rate $v$ and

$$
\delta^{*}=\sqrt{\frac{\nu \gamma}{M(N-1)} \sum_{q=1}^{N-1} \alpha_{1}^{-1}} .
$$

This is a highly interesting result since in the perfect escape case $(v=\infty)$ this minimum disappears, as $\delta^{*}$ is pushed toward $\infty$. Thus the "imperfect" escape window enables a region that was absent in the perfect case. On the other hand, in the limit $\beta \gg \delta$ it could be shown that Eq. (16) cannot be satisfied for any $\delta^{*}$ value. In this case, and taking into account the walker's initial distribution, the transport is performed on the baseline (lower boundary) of the confining domain, so this is an expected behavior.
In the following section we will make more remarks regarding the minimum in $T$, while introducing some illustrations corresponding to $\delta^{*}$.

\section{ILLUSTRATIONS}

Here we illustrate the general framework introduced in the previous section and compare our theoretical results to independent Monte Carlo simulations. A brief review of our simulation methodology is appropriate at this point. Basically we follow a continuous time random walk (CTRW) [22] simulation scheme while the theory follows a master equation approach. We uniformly distribute the searchers on the baseline [except on the escape window, which is placed at $(x, y)=(0,0)]$ of a two-dimensional $N \times(M+1)$ lattice with periodic boundary conditions. The propagation of the walkers in the presence of the "imperfect" escape window is implemented as follows: each walker is independent of the others (and we have used $10^{6}$ of them) and is assigned an internal clock that is updated according to its waiting time probability distribution. We define an indicator function that records the needed information: if the window was "crossed" (the walker left the system) and the time in which this happened. The walker's jump probabilities are evaluated in terms of the transition rates: on the surface $(\gamma)$, on the baseline - but not in the escape window- $(\beta, \delta)$, and in the escape window $(\beta, \delta, \nu)$. We check whether the trapping conditions are fulfilled (for each walker), and if they are, we update our indicator function. If the trapping conditions are not fulfilled, the dynamics continues until trapping is achieved. Once all walkers succeed in crossing the window the averaged indicator function produces the output of interest, the MET.

In the next figures, lines indicate analytical calculations while symbols correspond to Monte Carlo (MC) simulations. We will be interested in situations in which a mixed type of transport generates a global minimum in the mean escape time.

In Fig. 3 we present curves corresponding to the MET as a function of the desorption rate $\delta$, with parameters $N=20, M=10$, and $\beta=0.1$, for different values of the escape "strength" $\nu$, which is the "transition rate" at the escape window. We have included for comparison the "perfect escape case," i.e., once in the escape window the escape is instantaneous, with no dwelling time. Notice how $v$ regulates the existence of a minimum in the MET: as $v$ gets smaller the imperfection raises the $T$ curve until it becomes monotonic. Hence, for this situation we could say that $v$ affects negatively the "mixed" type of transport (journey's along the boundaries and the surface). However, it is worth remarking that the transition rate at the escape window can contribute positively as well. This behavior is well depicted in Fig. 4.

Figure 4 presents curves corresponding to the MET as a function of the desorption rate $\delta$, with $N=10, M=2$, and $\beta=0.01$, for different values of the transition rate $v$. As can be inferred from the figure, $v$ significantly influences the MET as it varies from 0 to $\infty$. Changes in the location of the extrema values of the MET can be seen ranging from a monotonic behavior $(\nu \rightarrow \infty$ extrema in $\delta \rightarrow \infty)$ to a situation with a global minimum, and then back again into a monotonic behavior ( $\nu \rightarrow 0$ extrema in $\delta \rightarrow 0$ ). So in this case the transition rate to the escape window contributes "positively" 


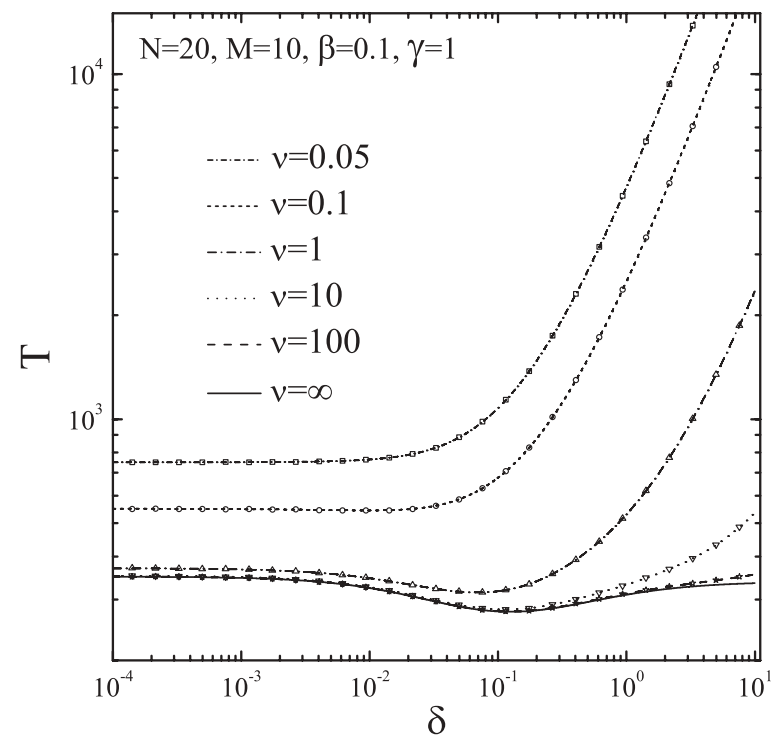

FIG. 3. MET as a function of the desorption rate $\delta$ (on a log scale), with $M=10, N=20$, and $\beta=0.1$, for different values of the transition rate at the escape window $v$. From top to bottom $v=0.05$, $0.1,1,10$, and 100 . For comparison, we have also included the perfect escape window case $(v=\infty)$ (thick solid line).

to the mixed type of transport, since it turns a situation without a minimum (perfect escape case) into a situation of enhanced transport (minimum in $T$ for some values of $v$ ).

In Fig. 5 we present curves corresponding to the $\delta$ value that minimizes MET, $\delta^{*}$, as a function of $\beta$ for different values of $v$, obtained from the numerical solution of Eq. (16). All lines depict quite a similar trend for finite $v ; \delta^{*}\left(\beta_{\circ}, N, M, \gamma, v\right)=0$ values marked by empty circles are not included in the curves and mark the end of the $\beta$ interval in which $\delta^{*}$ exists. In other words $T$ is not monotonic while $\beta \in\left[0, \beta_{\circ}\right)$. As we show in

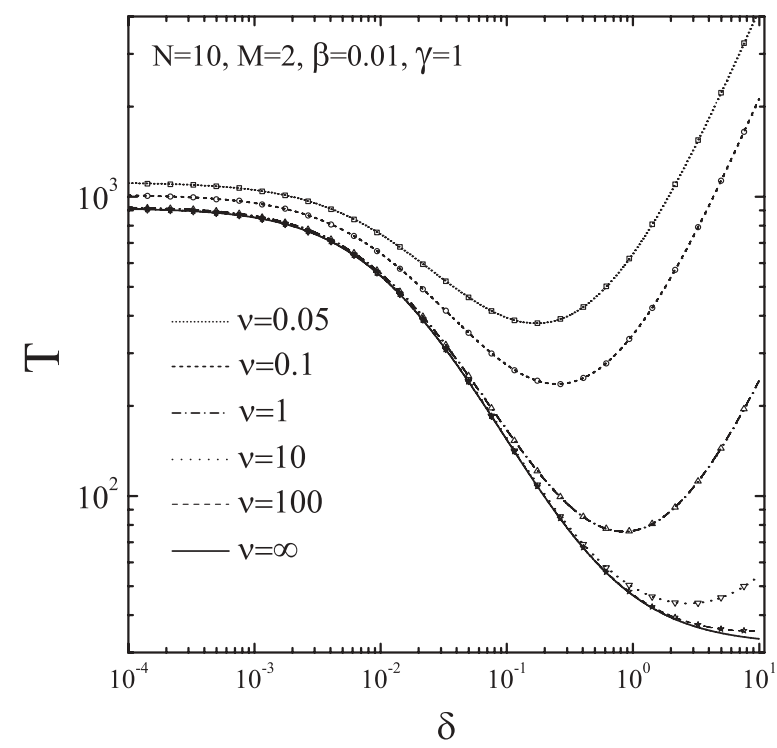

FIG. 4. MET as a function of the desorption rate $\delta$ (on a log-log scale), with $\beta=0.01, N=10$, and $M=2$, for different values of $v$ (transition rate at the escape window). From bottom to top $v=\infty$, $100,10,1,0.1$, and 0.05 . Lines correspond to analytical calculations and symbols to Monte Carlo simulations.

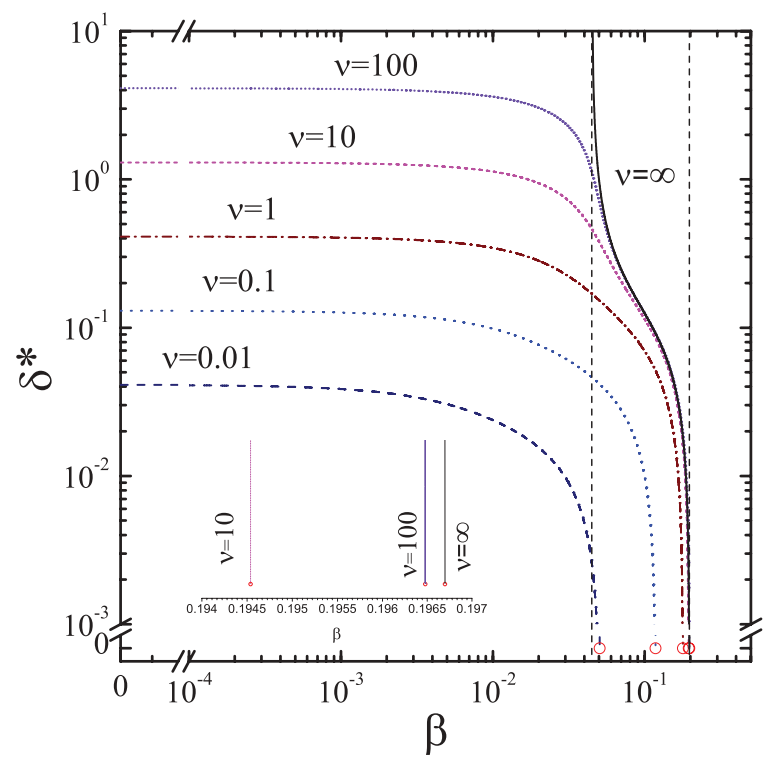

FIG. 5. (Color online) $\delta^{*}$ as a function of $\beta$ (on a log-log scale) for fixed $N, M$, and $\gamma$ for different values of $\nu$. The cuts of $\delta^{*}$ on the $\beta$ axis $\left[\delta^{*}\left(\beta_{\circ}\right)=0\right]$ for different $\nu$, indicated by red empty circles, are not included in the curve; $\beta$ 。 points are obtained from Eq. (18). Vertical dashed lines are the asymptotes for the perfect escape case obtained from Eqs. (19) and (20).

Appendix $\mathrm{C} \beta_{\circ}$ satisfies

$$
2 \beta_{\circ}=\frac{\gamma}{M} \frac{\sum_{q=1}^{N-1} \alpha_{1} \alpha_{2}^{-2}}{\frac{(N-1) 2 \beta_{\circ}}{v}+\sum_{q=1}^{N-1} \alpha_{2}^{-1}} .
$$

For values larger than $\beta_{\circ}$ the $T$ curve reaches a minimum at $\delta=0$. However, this is found at the beginning of the $\delta$ interval and without change of sign of $\partial T / \partial \delta$. We decided to rule it out as long as it does not represent a true interplay between surface and boundary paths. In these situations all particles stay on the baseline and eventually escape trough the escape window without excursions into the surface.

The behavior of $\delta^{*}$ considerably changes in the perfect case $(v=\infty)$. Particularly, the range of $\beta$ values where a minimum exists in $T$ shrinks, as indicated by the dashed asymptotes in the figure. The left and right asymptotes indicate the limit in which $T$ becomes monotonic, extrema for $\delta^{*} \rightarrow \infty$ and $\delta^{*} \rightarrow$ 0 , respectively. The left and right asymptotes are, respectively, located at

$$
\begin{aligned}
& 2 \beta_{\delta^{*} \rightarrow \infty}=\frac{\gamma}{M} \sum_{q=1}^{N-1} \alpha_{1}^{-1}\left(\sum_{q=1}^{N-1} \alpha_{2} \alpha_{1}^{-2}\right)^{-1}, \\
& 2 \beta_{\delta^{*} \rightarrow 0}=\frac{\gamma}{M} \sum_{q=1}^{N-1} \alpha_{1} \alpha_{2}^{-2}\left(\sum_{q=1}^{N-1} \alpha_{2}^{-1}\right)^{-1}
\end{aligned}
$$

For clarity sake in the inset we have magnified the entry points to the $\beta$ axis of $\delta^{*}$ curves for $\nu=10,100$, and $\infty$.

Figure 6 shows the phase diagrams that summarize the existence and nonexistence of enhanced transport, analyzed from the perspective of the existence of a minimum in the mean escape time. The diagrams are plotted for fixed $N$, $M$, and $v$ as a function of the transition probability over 


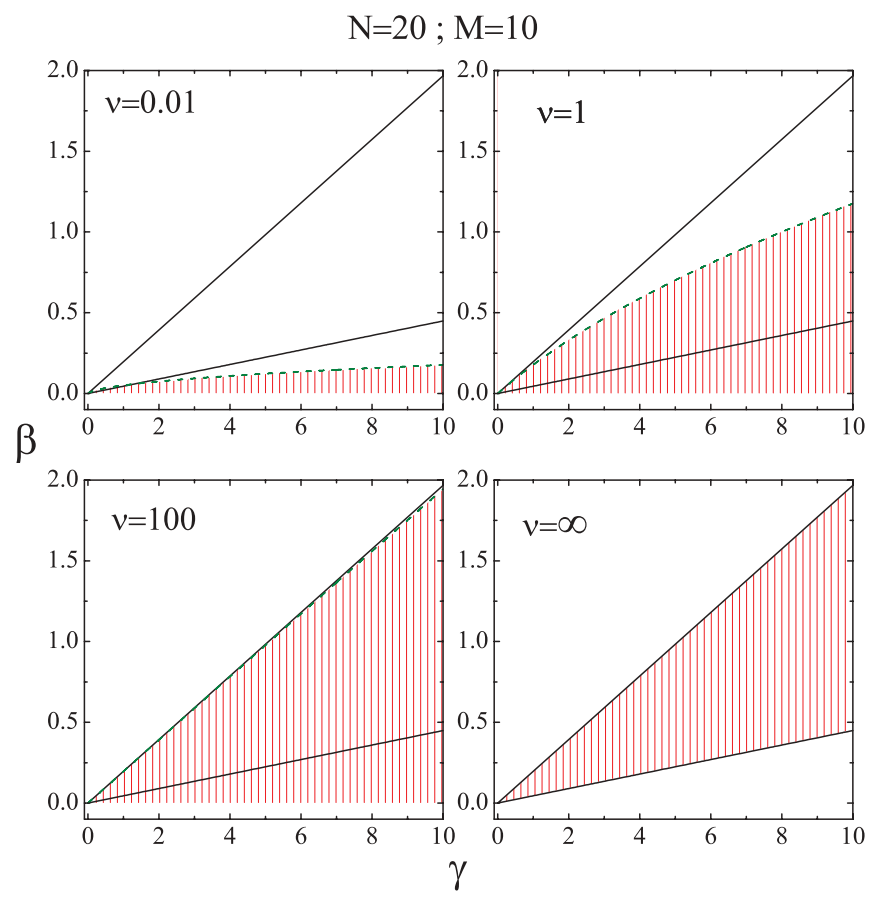

FIG. 6. (Color online) Phase diagrams that summarize the existence and nonexistence of enhanced transport for $v=0.01,1,100$, and $\infty$, respectively, for fixed system sizes $N=20$ and $M=10$. White regions correspond to nonoptimal transport, while filled regions (red patterns) identify regimes of enhanced transport. Regions enclosed by black lines correspond to enhanced transport in the perfect trapping case $(v=\infty)$ while dashed (green) lines correspond to the bound from Eq. (18), after which $T$ becomes monotonic.

the baseline, $\beta$, and the surface transition probability $\gamma$. White regions correspond to nonoptimal transport (absence of minimum-monotonic — in the MET), while filled regions (red patterns) identify regimes with enhanced transport. We have also included in Fig. 6, enclosed by black lines, the region corresponding to enhanced transport in the perfect window escape case. Dashed curves are obtained from the solution of Eq. (18) with $\beta_{\circ}>0$,

$$
2 \beta_{\circ}=-\frac{v \sum_{q} \alpha_{2}^{-1}}{2(N-1)}+\sqrt{\gamma \frac{v \sum_{q} \alpha_{2}^{-1}}{M(N-1)}+\left(\frac{v \sum_{q} \alpha_{1} \alpha_{2}^{-2}}{2(N-1)}\right)^{2}} .
$$

As expected, when $v$ grows the regions approach the perfect case and the escape, once in the window, becomes certain and instantaneous. Notice that we obtain quite a good agreement between the region of optimal transport, evaluated from Eq. (14), and the corresponding bounds derived from relations (21) for finite $v$ and (19) and (20) for $v=\infty$.

In Fig. 7 we include the relative error $[\epsilon=\sqrt{\operatorname{var}(T)} / T]$ of the MET as a function of the starting position of the walker for different values of $\delta$ and $v$ obtained through MC simulations for system parameters $N=20, M=10$, and $\beta=0.1$. In this figure we only show $s_{0}=1, \ldots, 10$ [with the escape window located at $(0,0)]$ since $s_{0}=11, \ldots, 19$ produces the same output due to the symmetry imposed by the boundary conditions. We observe that $\epsilon$ depends on the starting position $\left(s_{0}\right)$ and the $v$ parameter. The $s_{0}$ dependence would indicate that this may be considered as an additional parameter in the MET optimization. Figures 7(a) and 7(b) show that when $\epsilon>1$ the MET may be an insufficient measure for characterizing the escape process [31] while when $\epsilon<1$ the MET is a suitable magnitude. In the former case $(\epsilon>1)$ other magnitudes, e.g., the coverage territory [21], may be considered in order to fully characterize the efficiency of the mixed type of transport.

\section{CONCLUSIONS}

We have presented a model based on a master equation approach to the narrow-escape-time problem. In this study we introduced a finite transition probability, $v$, at the narrow escape window which allowed us to study the imperfect escape
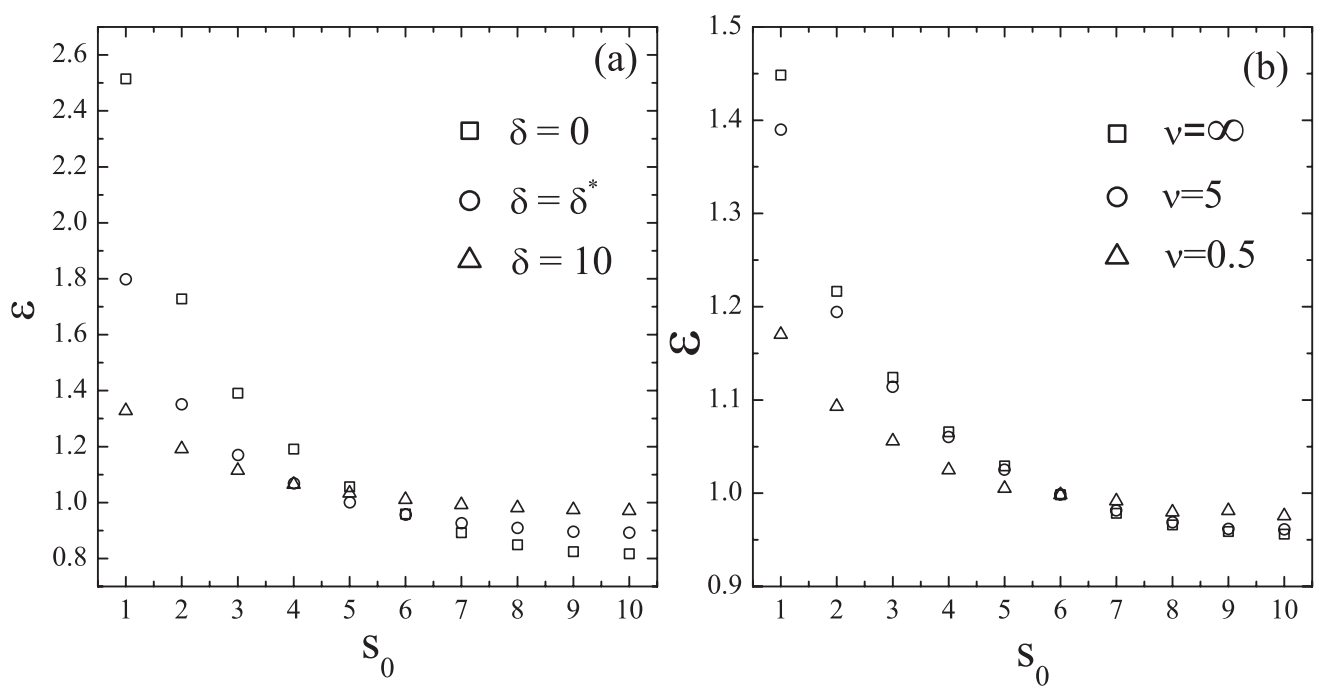

FIG. 7. (a) Relative error as a function of the starting position for different values of $\delta$ and $v=\infty$. (b) Relative error as a function of the starting position for different values of $v$ and $\delta=1$. All points corresponds to system parameters $N=20, M=10$, and $\beta=0.1$. All data correspond to MC simulation. 
case. Varying from 0 to $\infty, v$ allowed the study of both extremes of the trapping process: that of a highly deficient capture and situations where escape is certain (perfect trapping case).

By resorting to Dyson's technique we have obtained analytic results for the primary quantity studied in the NET problem, the mean escape time, and we have studied its dependence in terms of the transition (desorption) probability over (from) the surface boundary, the confining domain dimensions, and the finite transition probability at the escape window. Particularly, we showed that the existence of a global minimum in the NET is controlled by the "imperfection" of the escape process. A very interesting result was that the "imperfect" escape window enabled a region where $T$ could be minimized, something the perfect case lacked.

We have also presented bounds-Eqs. (18), (19), and (20)_between which an optimal minimum value of $T$ could be found, improving previous bounds derived in [19]. The phase diagrams introduced in the last section deserve a special word, for not only do they present a compact summary of the situations of enhanced transport, whenever some exist, but they also can lead to a better understanding of the relations among the parameters that characterize the system. In addition to our analytical approach, we have implemented Monte Carlo simulations, finding excellent agreement between the theoretical results and simulations.

We consider that the presented scheme is an analytically manageable model, which could be used to study the impact of several (domain dimension, different rates of transition, etc.) parameters in the interplay between surface and boundary pathways and could also serve as a forerunner for the study of more general and complex systems. This work contributes to an area of growing interest, providing a more general overview of a previous work [19] and showing a plausible physical insight into the surface-mediated diffusion mechanisms in the presence of an imperfect escape window.

The current approach to the narrow-escape-time problem can be generalized in several directions: higher dimensions, dynamical behavior of the narrow escape window, nonMarkovian desorption, etc. All of these aspects will be the subject of future work.

\section{ACKNOWLEDGMENTS}

The authors thank C. E. Budde, Jr. for technical assistance. Support by CONICET and SeCyT (Universidad Nacional de Córdoba), Argentina, is acknowledged. HSW acknowledges financial support from MICINN, Spain, through Project No. FIS2010-18023.

\section{APPENDIX A: ABSORPTION AT VISIT $j$, THE $A_{j}(\cdot)$ INTERPRETATION}

By using the convolution property of Laplace transforms [32], Eq. (6) can be expressed in the temporal domain as

$$
\begin{aligned}
A_{j}\left(\overrightarrow{0}, t \mid \vec{s}_{0}, 0\right)= & \int_{0}^{t} \mathcal{L}^{-1}\left\{\frac{v}{\Psi(\overrightarrow{0}, u)^{-1}+v}\right\}\left(t-t^{\prime}\right) d t^{\prime} \\
& \times \int_{0}^{t^{\prime}} \Omega_{j-1}\left(t^{\prime}-t^{\prime \prime}\right) F\left(\overrightarrow{0}, t^{\prime \prime} \mid \vec{s}_{0}, 0\right) d t^{\prime \prime}
\end{aligned}
$$

(with the time order $0<t^{\prime \prime} \leqslant t^{\prime} \leqslant t$ ), where

$\Omega_{j-1}(t)=\mathcal{L}^{-1}\left\{\left(F(\overrightarrow{0}, u \mid \overrightarrow{0}, 0) \frac{\Psi(\overrightarrow{0}, u)^{-1}}{\Psi(\overrightarrow{0}, u)^{-1}+v}\right)^{j-1}\right\}(t)$

accounts for the $(j-1)$ returns until the walker is trapped. Let us focus on $\Omega_{j-1}(t)$; for example, we set $j=1$, so in this case $\Omega_{0}(t)=\mathcal{L}^{-1}\{1\}(t)=\delta\left(t^{+}\right)$(Dirac's delta function) and get

$$
\begin{aligned}
A_{1}\left(\overrightarrow{0}, t \mid \vec{s}_{0}, 0\right)= & \int_{0}^{t} \mathcal{L}^{-1}\left\{\frac{v}{\Psi(\overrightarrow{0}, u)^{-1}+v}\right\}\left(t-t^{\prime}\right) d t^{\prime} \\
& \times \int_{0}^{t^{\prime}} \delta\left(t^{\prime}-t^{\prime \prime}\right) F\left(\overrightarrow{0}, t^{\prime \prime} \mid \vec{s}_{0}, 0\right) d t^{\prime \prime} \\
= & \int_{0}^{t} \mathcal{L}^{-1}\left\{\frac{v}{\Psi(\overrightarrow{0}, u)^{-1}+v}\right\}\left(t-t^{\prime}\right) F\left(\overrightarrow{0}, t^{\prime} \mid \vec{s}_{0}, 0\right) d t^{\prime} ;
\end{aligned}
$$

i.e., the walker arrives at the origin at $t^{\prime}$ for the first time and is trapped between $t^{\prime}$ and $t$ in its first visit; for $j=2$ we get

$$
\begin{aligned}
A_{2}\left(\overrightarrow{0}, t \mid \vec{s}_{0}, 0\right)= & \int_{0}^{t} \mathcal{L}^{-1}\left\{\frac{v}{\Psi(\overrightarrow{0}, u)^{-1}+v}\right\}\left(t-t^{\prime}\right) d t^{\prime} \\
& \times \int_{0}^{t^{\prime}} \Omega_{1}\left(t^{\prime}-t^{\prime \prime}\right) F\left(\overrightarrow{0}, t^{\prime \prime} \mid \vec{s}_{0}, 0\right) d t^{\prime \prime},
\end{aligned}
$$

where

$$
\begin{aligned}
\Omega_{1}(t) & =\mathcal{L}^{-1}\left\{\left(F(\overrightarrow{0}, u \mid \overrightarrow{0}, 0) \frac{\Psi(\overrightarrow{0}, u)^{-1}}{\Psi(\overrightarrow{0}, u)^{-1}+v}\right)^{1}\right\}(t) \\
& =\int_{0}^{t} \mathcal{L}^{-1}\left\{\frac{\Psi(\overrightarrow{0}, u)^{-1}}{\Psi(\overrightarrow{0}, u)^{-1}+v}\right\}(t-\tau) F(\overrightarrow{0}, \tau \mid \overrightarrow{0}, 0) d \tau
\end{aligned}
$$

is the probability of a single return until time $t$ with out trapping. So, $A_{2}(\cdot)$ involves a first visit at $t^{\prime \prime}$, makes a unique return between $t^{\prime \prime}$ and $t^{\prime}$, and finally gets trapped between $t^{\prime}$ and $t$. For the general term $A_{j}(\cdot)$, notice that the probability $\Omega_{j-1}(t)$ (for $j \geqslant 2$ ) could be written as

$$
\begin{aligned}
\Omega_{j-1}(t) & =\mathcal{L}^{-1}\left\{\Omega_{j-1}(u)\right\}=\mathcal{L}^{-1}\left\{\left(\Omega_{1}(u)\right)^{j-1}\right\} \\
& =\mathcal{L}^{-1}\left\{\Omega_{1}(u)\left(\Omega_{1}(u)\right)^{j-2}\right\} \\
& =\int_{0}^{t} \Omega_{1}(t-\tau) \mathcal{L}^{-1}\left\{\left(\Omega_{1}(u)\right)^{j-2}\right\}(\tau) d \tau .
\end{aligned}
$$

In the last line of the equation the second factor in the integral accounts for the $(j-2)$ returns to the origin without trapping until time $\tau$ and the first factor gives the last return between $\tau$ and $t$, i.e., $(j-1)$ returns until time $t$. Following similar reasoning (to $j=1,2$ ) and by iterating the convolution property of Laplace transforms on Eq. (A4), we are able to construct the whole hierarchy of return probabilities in terms of the first return probability $\Omega_{1}(t)$ while we get a clear physical interpretation of the $A_{j}(\cdot)$ terms.

\section{APPENDIX B: MET CALCULATION}

In this Appendix we focus on the calculation of the probability $P\left(n, m, t \mid n_{0}, m_{0}, t=0\right)$, which is the building block for the mean escape time. Taking the (finite) Fourier transform with respect to the $x$ variable and the Laplace transform with 
respect to the time $t$ in Eq. (9), we obtain

$$
\begin{aligned}
& m=0, \\
& u P(k, 0, u)-P(k, 0, t=0) \\
& =\gamma P(k, 1, u)-\left(\delta-A_{1}(k)\right) P(k, 0, u) \text {; } \\
& m=1 \text {, } \\
& u P(k, 1, u)-P(k, 1, t=0) \\
& =\delta P(k, 0, u)+\gamma P(k, 2, u)-(2 \gamma-A(k)) P(k, 1, u) ; \\
& 2 \leqslant m \leqslant M-1 \text {, } \\
& u P(k, m, u)-P(k, m, t=0) \\
& =A(k) P(k, m, u)+\gamma(P(k, m+1, u) \\
& +P(k, m-1, u)-2 P(k, m, u)) \text {; } \\
& m=M \\
& u P(k, M, u)-P(k, M, t=0) \\
& =A(k) P(k, M, u)+\gamma P(k, M-1, u)-\gamma P(k, M, u) \text {. }
\end{aligned}
$$

Here we have defined $A_{1}(k)=2 \beta(\cos k-1)$ and $A(k)=$ $2 \gamma(\cos k-1)$. By using the matrix formalism, Eq. (B1) can be written as

$$
[u \mathbb{I}-\mathbb{H}] \mathbb{P}=\mathbb{I},
$$

where $\mathbb{I}$ is the identity matrix, $\mathbb{H}$ is an $(M+1) \times(M+1)$ tridiagonal matrix with elements

$$
\mathbb{H}=\left[\begin{array}{cccccc}
C_{1} & \gamma & 0 & \ldots & \ldots & 0 \\
\delta & C & \gamma & 0 & \ldots & 0 \\
0 & \gamma & C & \gamma & 0 & \vdots \\
\cdots & 0 & \ddots & \ddots & \ddots & \vdots \\
\cdots & \ldots & \ldots & \gamma & C & \gamma \\
0 & \ldots & & 0 & \gamma & \gamma+C
\end{array}\right],
$$

$C$ and $C_{1}$ are defined as $C=-2 \gamma+A(k)$ and $C_{1}=-\delta+$ $A_{1}(k)$, and $\mathbb{P}$ is an $(M+1) \times(M+1)$ matrix with components

$$
[\mathbb{P}(k, u)]_{m, m_{0}}=P\left(k, m, u \mid n_{0}, m_{0}, t=0\right) .
$$

In order to find the solution to Eq. (B2) we decompose the $\mathbb{H}$ matrix in the following way:

$$
\mathbb{H}=A(k) \mathbb{I}+\mathbb{H}^{0}+\mathbb{H}^{1}+\mathbb{H}^{2},
$$

where

$$
\mathbb{H}^{0}=\left[\begin{array}{ccccc}
-\gamma & \gamma & 0 & . . & 0 \\
\gamma & -2 \gamma & \gamma & . . & 0 \\
0 & \gamma & -2 \gamma & \gamma & 0 \\
. . & . . & . . & . . & \\
. . & . . & \gamma & -2 \gamma & \gamma \\
. \cdot & & 0 & \gamma & -\gamma
\end{array}\right]
$$

corresponds to the transition matrix for a symmetric random walk to nearest neighbors in a finite lattice $(M+1$ sites) with reflective boundary conditions at the ends. On the other hand,

$$
\mathbb{H}^{1}=\left(\gamma-\delta+A_{1}(k)-A(k)\right) \delta_{i, 0} \delta_{0, j}=\Delta_{1} \delta_{i, 0} \delta_{0, j},
$$

$$
\mathbb{H}^{2}=-(\gamma-\delta) \delta_{i, 1} \delta_{0, j}=\Delta_{2} \delta_{i, 1} \delta_{0, j}
$$

A formal solution to Eq. (B2) is

$$
\mathbb{P}=[u \mathbb{I}-\mathbb{H}]^{-1} .
$$

By applying the Dyson procedure [26] a general expression in the Fourier-Laplace space for $[\mathbb{P}(k, u)]_{m, m_{0}}$ can be found:

$$
\begin{aligned}
{[\mathbb{P}(k, u)]_{m, m_{0}} } \\
=\left[\mathbb{P}^{0}(k, u)\right]_{m, m_{0}}+\left[\mathbb{P}^{0}(k, u)\right]_{m, 0}\left[\mathbb{P}^{0}(k, u)\right]_{0, m_{0}} \\
\quad \times \frac{\Delta_{1}}{1-\Delta_{1}\left[\mathbb{P}^{0}(k, u)\right]_{0,0}}+\frac{\left[\mathbb{P}^{0}(k, u)\right]_{0, m 0} \cdot \Delta_{2}}{1-\left(\Delta_{1}+\Delta_{2}\right)\left[\mathbb{P}^{0}(k, u)\right]_{0,0}} \\
\quad \times\left(\left[\mathbb{P}^{0}(k, u)\right]_{m, 1}+\frac{\left[\mathbb{P}^{0}(k, u)\right]_{m, 0}\left[\mathbb{P}^{0}(k, u)\right]_{0,1} \cdot \Delta_{1}}{1-\Delta_{1}\left[\mathbb{P}^{0}(k, u)\right]_{0,0}}\right),
\end{aligned}
$$

where

$$
\begin{aligned}
{\left[\mathbb{P}^{0}(k, u)\right]_{m, m_{0}} } & =\frac{\eta^{\left|m-m_{0}\right|}+\eta^{\tilde{M}-\left(m+m_{0}\right)}}{2 \gamma(1-\eta)+\tilde{u}}, \\
\tilde{u} & =u-A(k), \quad \tilde{M}=2 M+1, \quad \text { and } \\
\eta & =1+\left(\tilde{u}-\sqrt{\tilde{u}^{2}+4 \gamma \tilde{u}}\right) / 2 \gamma .
\end{aligned}
$$

From (B9), the probability that a walker is at site $(n, m)$ at time $t$ given it was at $\left(n_{0}, m_{0}\right)$ at $t=0, P\left(n, m, t \mid n_{0}, m_{0}, t=0\right)$, is derived by using the inverse Laplace transform on $u$ and the inverse Fourier transform on $k$ (for the $x$ coordinate) for each matrix element $[\mathbb{P}(k, u)]_{m, m_{0}}$. Notice that, as we are interested in the calculation of (8), we only need to perform the inverse Fourier transform on $P\left(0,0, u \mid n_{0}, m_{0}, t=0\right)$; i.e., we need the elements $\mathcal{F}^{-1}\left\{[\mathbb{P}(k, u)]_{0, m_{0}}\right\}$. In this case expression (B9) reduces to

$[\mathbb{P}(k, u)]_{0, m_{0}}=\frac{\eta^{m_{0}}+\eta^{\tilde{M}-m_{0}}}{\delta(1-\eta)\left(1-\eta^{\tilde{M}-1}\right)+\left[u-A_{1}(k)\right]\left(1+\eta^{\tilde{M}}\right)}$.

The inverse Fourier transform on $[\mathbb{P}(k, u)]_{0, m_{0}}$ is carried out in the following way:

$$
P\left(0,0, u \mid n_{0}, m_{0}, t=0\right)=\frac{1}{N} \sum_{q=0}^{N-1} e^{i \frac{2 \pi n_{0} q}{N}}\left[\mathbb{P}\left(\frac{2 \pi q}{N}, u\right)\right]_{0, m_{0}} .
$$

Thus we have obtained all the required expressions for the calculation of the MET. We now proceed to evaluate the mean escape time for a walker with an uniform initial distribution on the baseline $(y=0)$, i.e., $g(n, m)=\left(1-\delta_{n, 0}\right) \delta_{m, 0} /(N-1)$. Notice that we explicitly exclude the possibility of having a walker at $(0,0)$ at $t=0$. We obtain

$$
T=N\left[\frac{M}{\gamma}+\frac{1}{\delta}\right]\left\{\frac{\delta}{v}+\frac{\delta}{N-1} \sum_{q=1}^{N-1}\left[\mathbb{P}\left(\frac{2 \pi q}{N}, u=0\right)\right]_{0,0}\right\}
$$


or

$$
T=N\left[\frac{M}{\gamma}+\frac{1}{\delta}\right]\left\{\frac{\delta}{v}+\frac{\delta}{N-1} \sum_{q=1}^{N-1} \frac{1}{\alpha_{1} \delta+\alpha_{2} 2 \beta}\right\},
$$

where

$$
\begin{gathered}
\alpha_{1}=\alpha_{1}(q, N, M)=\frac{\left(1-\eta_{u=0}\right)\left(1-\eta_{u=0}^{2 M}\right)}{\left(1+\eta_{u=0}\right)^{2 M+1}}, \\
\alpha_{2}=\alpha_{2}(q, N)=1-\cos \frac{2 \pi}{N} q .
\end{gathered}
$$

\section{APPENDIX C: $\delta^{*}$, OPTIMAL DESORPTION PROBABILITY}

In this Appendix we present some results regarding the desorption probability rate value that minimizes $T, \delta^{*}$. For this recall Eq. (16),

$$
\left.\frac{\partial T}{\partial \delta}\right|_{\delta=\delta^{*}}=\frac{N M}{\gamma \nu}+\frac{N}{N-1} \sum_{q=1}^{N} \frac{M \gamma^{-1} 2 \beta \alpha_{2}-\alpha_{1}}{\left(\alpha_{1} \delta^{*}+\alpha_{2} 2 \beta\right)^{2}}=0 .
$$

Let us focus on the relation between $\delta$ and $\beta$ as these are the parameters of interest, since the former enables the transport on the surface, and the latter regulates the movement on the boundary line where the escape window is located. Although we will keep track of all variables, it could be shown that $(\mathrm{C} 1)$ can be written in terms of the scaled variables $\beta^{\prime}=\beta \gamma^{-1}$, $\nu^{\prime}=v \gamma^{-1}$, and $\delta^{\prime}=\delta \gamma^{-1}$. So a modification in $\gamma$ would result in an enlargement or shrinkage (if we let $\gamma$ get smaller or larger, respectively) of the former variables. We will consider the behavior of $\partial \delta^{*} / \partial \beta$. Even though we were not able to obtain an explicit expression for $\delta^{*}$, its derivative with respect to $\beta$ could be evaluated in closed form. To do this recall Eq. (C1) and differentiate it with respect to $\beta$ by considering $\delta^{*}=f(\beta)$ (with the other parameters held fixed). Then after some algebra we obtain

$$
\frac{\partial \delta^{*}}{\partial \beta}=\frac{\sum_{q=1}^{N-1} \frac{2\left(M \gamma^{-1} \delta^{*}+1\right) \alpha_{1} \alpha_{2}}{\left(\alpha_{1} \delta^{*}+\alpha_{2} 2 \beta\right)^{3}}-\sum_{q=1}^{N-1} \frac{M \gamma^{-1} \alpha_{2}}{\left(\alpha_{1} \delta^{*}+\alpha_{2} 2 \beta\right)^{2}}}{\sum_{q=1}^{N-1} \frac{\left(M \gamma^{-1} \delta^{*}+1\right) \alpha_{1}^{2}}{\left(\alpha_{1} \delta^{*}+\alpha_{2} 2 \beta\right)^{3}}-\sum_{q=1}^{N-1} \frac{M \gamma^{-1} \alpha_{1}}{\left(\alpha_{1} \delta^{*}+\alpha_{2} 2 \beta\right)^{2}}} .
$$

From (C2) we could obtain the entry points to the $\beta$ axis of $\delta^{*}$ curves. The forerunner for this is the sharp growth on the $\delta^{*}$ curves in Fig. 5. As a matter of fact the abrupt increase is in $\partial \log _{10} \delta^{*} / \partial \log _{10} \beta$. However, it is not difficult to show that this happens only if the denominator in (C2) $\rightarrow 0$ for some $\beta$ 。 (also notice that in this situation $\delta^{*} \sim 0$ ), so

$$
\begin{aligned}
\sum_{q=1}^{N-1} \frac{\alpha_{1}^{2}}{\left(\alpha_{2} 2 \beta_{\circ}\right)^{3}} & =M \gamma^{-1} \sum_{q=1}^{N-1} \frac{\alpha_{1}}{\left(\alpha_{2} 2 \beta_{\circ}\right)^{2}} \\
& =\left(M \gamma^{-1}\right)^{2}\left(\frac{(N-1)}{v}+2 \beta_{\circ} \sum_{q=1}^{N-1} \frac{\alpha_{1}}{\left(\alpha_{2} 2 \beta_{\circ}\right)^{2}}\right),
\end{aligned}
$$

where we have used $(\mathrm{C} 1)$ to replace the sum on the right-hand side. We could go even further and replace the sum on the left-hand side. For this we differentiate $(\mathrm{C} 1)$ with respect to $\delta^{*}$ and get $M \gamma^{-1} 2 \beta \sum_{q} \alpha_{1} \alpha_{2}\left(\alpha_{1} \delta^{*}+\alpha_{2} 2 \beta\right)^{-3}=\sum_{q} \alpha_{1}^{2}\left(\alpha_{1} \delta^{*}+\right.$ $\left.\alpha_{2} 2 \beta\right)^{-3}$. By using this relation and rearranging some terms in $(\mathrm{C} 3)$ we finally obtain

$$
2 \beta_{\circ}=\frac{\gamma}{M} \frac{\sum_{q=1}^{N-1} \alpha_{1} \alpha_{2}^{-2}}{\frac{(N-1) 2 \beta_{\circ}}{v}+\sum_{q=1}^{N-1} \alpha_{2}^{-1}} .
$$

The solution of Eq. (C4) in terms of $\beta_{\circ}$ that makes $\delta^{*}\left(\beta_{\circ}, N, M, \gamma, \nu\right)=0$ marks the end of the interval in which $\delta^{*}$ exists, i.e., the $T$ curve becomes monotonic.

For the perfect escape case we obtain one of the asymptotes between which $\delta^{*}$ exits, outside of which the extrema is pushed either to 0 or to $\infty$, by letting $v \rightarrow \infty$ in (C4):

$$
2 \beta_{\delta^{*} \rightarrow 0}=\frac{\gamma}{M} \frac{\sum_{q=1}^{N-1} \alpha_{1} \alpha_{2}^{-2}}{\sum_{q=1}^{N-1} \alpha_{2}^{-1}} .
$$

For the second asymptote we go back to (C2), follow similar reasoning that led us to Eq. (C3) (here $v=\infty$ and $\delta^{*} \rightarrow \infty$ ), and obtain

$$
2 \beta_{\delta^{*} \rightarrow \infty}=\frac{\gamma}{M} \frac{\sum_{q=1}^{N-1} \alpha_{1}^{-1}}{\sum_{q=1}^{N-1} \alpha_{2} \alpha_{1}^{-2}} .
$$

Equations (C5) and (C6) constitute an improvement to the bounds derived in [19] and the solution of Eq. (C4) gives a bound regarding the existence of a minimum in the MET.
[1] O. Bénichou and R. Voituriez, Phys. Rev. Lett. 100, 168105 (2008).

[2] G. Tkačik and W. Bialek, Phys. Rev. E 79, 051901 (2009).

[3] M. A. Lomholt, B. van den Broek, S.-M. J. Kalisch, G. J. L. Wuite, and R. Metzler, Proc. Natl. Acad. Sci. USA 106, 8204 (2009).

[4] O. Bénichou, C. Loverdo, and R. Voituriez, Europhys. Lett. 84, 38003 (2008).

[5] M. G. E. da Luz, A. Grosberg, E. P. Raposo, and G. M. Viswanathan, J. Phys. A: Math. Theor. 42, 430301 (2009).

[6] P. G. de Gennes, Adv. Colloid Interface Sci. 27, 189 (1987).

[7] J. F. Douglas, H. E. Johnson, and S. Granick, Science 262, 2010 (1993).

[8] O. V. Bychuk and B. O’Shaughnessy, Phys. Rev. Lett. 74, 1795 (1995).
[9] J. H. Clint, Surfactant Aggregation (Chapman and Hall, New York, 1992).

[10] H. Berg and E. Purcell, Biophysical 20, 193 (1977).

[11] O. G. Berg, R. B. Winter, and P. H. Von Hippel, Biochemistry 20, 6929 (1981).

[12] J. Linderman and D. Lauffenburger, Biophysical 50, 295 (1986).

[13] H.-X. Zhou and R. Zwanzig, J. Chem. Phys. 94, 6147 (1991).

[14] I. V. Grigoriev, Y. A. Makhnovskii, A. M. Berezhkovskii, and V. Y. Zitserman, J. Chem. Phys. 116, 9574 (2002).

[15] A. Singer, Z. Schuss, D. Holcman, and R. Eisenberg, J. Stat. Phys. 122, 437 (2006); A. Singer, Z. Schuss, and D. Holcman, ibid. 122, 465 (2006); A. Singer and Z. Schuss, Phys. Rev. E 74, 020103 (2006). 
[16] P. C. Bressloff and B. A. Earnshaw, Phys. Rev. E 75, 041915 (2007).

[17] G. Oshanin, M. Tamm, and O. Vasilyev, J. Chem. Phys. 132, 235101 (2010).

[18] O. Bénichou, D. Grebenkov, P. Levitz, C. Loverdo, and R. Voituriez, Phys. Rev. Lett. 105, 150606 (2010); J. Stat. Phys. 142, 657 (2011).

[19] F. Rojo and C. E. Budde, Phys. Rev. E 84, 021117 (2011).

[20] R. Walder, N. Nelson, and D. K. Schwartz, Phys. Rev. Lett. 107, 156102 (2011).

[21] T. Calandre, O. Bénichou, D. S. Grebenkov, and R. Voituriez, Phys. Rev. E 85, 051111 (2012).

[22] J. W. Haus and K. W. Kehr, Phys. Rep. 150, 263 (1987).

[23] M. A. Ré and C. E. Budde, Phys. Rev. E 61, 1110 (2000).

[24] W. Nadler and D. L. Stein, J. Chem. Phys. 104, 1918 (1996).
[25] M. Coppey, O. Benichou, R. Voituriez, and M. Moreau, Biophys. J. 87, 1640 (2004); A. M. Berezhkovskii and A. V. Barzykin, J. Chem. Phys. 136, 054115 (2012).

[26] J. A. Revelli, C. E. Budde, and H. S. Wio, J. Phys.: Condens. Matter 17, S4175 (2005).

[27] J. L. Spouge, A. Szabo, and G. H. Weiss, Phys. Rev. E 54, 2248 (1996).

[28] A. J. F. Siegert, Phys. Rev. 81, 617 (1951); F. Rojo, P. A. Pury, and C. E. Budde, Physica A 389, 3399 (2010).

[29] See [22], and references therein.

[30] This consideration will help us in the comparison with the perfect escape case and avoids the "instantaneous" escaping in the limit $v \rightarrow \infty$.

[31] T. G. Mattos, C. Mejía-Monasterio, R. Metzler, and G. S. Oshanin, arXiv:1206.1003 [cond-mat.stat-mech].

[32] M. Abramowitz and I. Stegun, Handbook of Mathematical Functions (Dover, New York, 1965). 